\title{
Broadband detection of squeezed vacuum: A spectrum of quantum states
}

\author{
G. Breitenbach, F. Illuminati*, S. Schiller, and J. Mlynek \\ Fakultät für Physik, Universität Konstanz, D-78457 Konstanz, Germany \\ http://quantum-optics.physik.uni-konstanz.de
}

* Permanent address: Dipartimento di Fisica, Università di Salerno, and INFM, Unità di Salerno, I-84081 Salerno, Italy

Phone: +49(7531) 883842, FAX +49(7531) 883072, e-mail: Gerd.Breitenbach@uni-konstanz.de

We demonstrate the simultaneous quantum state reconstruction of the spectral modes of the light field emitted by a continuous wave degenerate optical parametric amplifier. The scheme is based on broadband measurement of the quantum fluctuations of the electric field quadratures and subsequent Fourier decomposition into spectral intervals. Applying the standard reconstruction algorithms to each bandwidth-limited quantum trajectory, a "spectrum" of density matrices and Wigner functions is obtained. The recorded states show a smooth transition from the squeezed vacuum to a vacuum state. In the time domain we evaluated the first order correlation function of the squeezed output field, showing good agreement with theory.

The experimental techniques of quantum state reconstruction, first applied five years ago, have opened a new field of research, wherein simple quantum mechanical systems can be characterized completely by density matrices and Wigner functions [1]. Our system consists of electromagnetic field modes at optical frequencies. We have previously generated the whole family of squeezed states of light using an optical parametric amplifier (OPA) and reconstructed these states using the method of optical homodyne tomography [2]. The reconstructions presented therein were limited to essentially one particular pair of modes at frequencies $\omega \pm \Omega$, where $\Omega$ is a radio frequency and $\omega$ the optical frequency. The spectral bandwidth of this mode pair was $\Delta \Omega / 2 \pi=100 \mathrm{kHz}$. Since an OPA pumped below threshold emits a frequency spectrum, with a bandwidth determined by the cavity linewidth in the order of several MHz, the output of the OPA is described more precisely by a whole spectrum of quantum states [3]. General schemes for multimode reconstruction become quite complicated already at the two-mode level [4]. So far only one experiment demonstrating photon- 
number correlations in the time domain by measuring the photon statistics via dual-pulse phaseaveraged homodyne detection has been carried out [5]. In this work we use a simple measurement scheme to record simultaneously the complete statistical information about the quantum states of all mode-pairs emitted by the OPA, disregarding correlations between the different mode-pairs. This enables us to reconstruct the Wigner functions and density matrices corresponding to a large set of mode pairs.

The experimental setup is shown in Fig. 1. Center piece of the experiment is a monolithic standing-wave lithium-niobate OPA, pumped by a frequency-doubled continuous-wave Nd:YAG laser $(532 \mathrm{~nm})$. The output of the OPA is analyzed by a homodyne detector whose difference current $i_{-}$is directly proportional to the OPA's output electric field. In order to obtain simultaneously information about the quantum states of all modes emitted by the OPA, the homodyne detector current $i_{-}$was recorded with a bandwidth covering the whole frequency range up to 30 $\mathrm{MHz}$, exceeding the OPA's 17.5 MHz cavity linewidth. For this purpose fast photodiodes (Epitaxx ETX500T, specified bandwidth $140 \mathrm{MHz}$ ) with broadband amplifiers and a $30 \mathrm{MHz}$ A/D-board (IMTEC) for data collection were employed. The $i_{-}$data (about 500000 points with 12 bit resolution) are taken while the local oscillator phase is swept by $2 \pi$ in approximately $8 \mathrm{~ms}$. The recorded noise trace is split into 16 separate traces, each containing the information of a mode pair of small bandwidth $1.9 \mathrm{MHz}$, centered at a sideband frequency $\omega \pm \Omega$. This is done by dividing the Fourier transform of the recorded trace into 16 equal intervals, and taking the inverse transform of each interval separately. The same is done for a measured $i_{-}$-trace of a vacuum state, in order to normalize each of the 16 traces. Due to electronic excess noise of the detection system at low frequencies the first of the 16 traces, containing the spectrum between 0 and $1.9 \mathrm{MHz}$, is discarded. The quantum states of the remaining 15 modes can be reconstructed the same way as described in [2], employing the inverse Radon transform [1] and the pattern functions of the harmonic oscillator [6]. Thus a whole "spectrum" of Wigner functions and density matrices is obtained from a single temporal record.

The main difference between the squeezed vacuum states reconstructed at different $\Omega$ is the amount of noise reduction (squeezing) and enhancement (antisqueezing). The spectra of quantum noise power of the squeezed quadrature $\Psi_{-}$and of the antisqueezed quadrature $\Psi_{+}$of an OPA on resonance are given by [3] 


$$
\Psi_{ \pm}(\Omega, P)=\Psi_{0}\left(1 \pm \xi \eta \frac{4 d}{(\Omega / \Gamma)^{2}+(1 \mp d)^{2}}\right)
$$

where $d=\sqrt{P / P_{t h}}$ is the pump parameter with pump power $P$ and a threshold power $P_{t h}$ of the OPA, $\Gamma / 2 \pi=17.5 \mathrm{MHz}$ is the cavity linewidth (HWHM), $\Psi_{0}$ is the spectral density of the vacuum state, $\xi=0.88$ is the escape efficiency of the resonator, and $\eta$ is the detection efficiency. The latter includes propagation losses after the OPA, homodyne efficiency and detector quantum efficiency. In contrast to our previous work we did not employ a mode cleaning cavity, thus classical noise of the pump wave was not negligible and the modematching between local oscillator and the OPA output signal was $\approx 95 \%$. Furthermore the efficiency of the photo detectors had degraded slightly from the previously measured 95-97\%, thus the overall detection efficiency including escape efficiency $\xi \cdot \eta$ was fitted to be 0.7 . The pump power was approximately half the threshold power. Fig.2 shows the spectra of the maximally squeezed and anti-squeezed quadratures measured directly with a spectrum analyzer in comparison with the ones obtained via multimode reconstruction.

Three of the reconstructed states are plotted in Fig.3. They display strongly elliptical Wigner functions and oscillations in the photon number [7], characteristics that vanish when the state aproaches a vacuum state at frequencies sufficiently away from the OPA cavity center frequency. We remark that we did not include error corrections in the reconstruction algorithm, as described in [8]. For a single mode treatment of previously measured data of us that takes into account the finite detection efficiency see [9]. Note also that the present measurement method does not allow to make any statements about possible correlations between the different mode-pairs. Regarding the light field generated by the OPA this is of minor importance, since according to theory mode-pairs at different frequencies should be uncorrelated [3].

A direct measure of the total noise of the squeezed and anti-squeezed quadratures $\int \Psi_{ \pm}(\Omega, P) d \Omega$ of the overall OPA output within the bandwidth 1.9-30 MHz is obtained by analyzing the full quantum noise instead of its particular Fourier components. (For this, the non-uniform spectral response of the A/D-board has to be eliminated via normalization in Fourier space.) The inset of Fig. 4 shows the total electric field variances as a function of the local oscillator phase. Well-known figures of this type usually depicted only the $E$-field variances of a single mode of the light field [10]. Here we obtain for the overall OPA output a total squeezing of $-2.9 \mathrm{~dB}(=0.47$ linear scale) and 6.7 $\mathrm{dB}(=4.7)$ for the total antisqueezing. The corresponding photon statistics is shown as well. This 
multimode light field would be detected if one would employ photon counters instead of homodyne detection. Note that no photon number oscillations are observable in the total OPA output. This may seem surprising, since almost all the photon number distributions of the individual modes show oscillations and all the reconstruction transformations are linear. However, according to basic laws of probability theory the overall sampled distribution of the quantum noise is not given by the average but by the convolution of the distributions of the individual modes. Therefore the photon statistics of the total OPA output is not given by averaging the single mode statistics. What is averaged though in our case of uncorrelated Gaussian noise distributions is, as mentioned above, the variance at each particular phase angle.

A further perspective is gained by the analysis of our data in the time domain. At a fixed phase $\theta$ of the local oscillator, the recorded time trace can be regarded as the quantum trajectory of the quadrature $x_{\theta}(t)=2^{-1 / 2}\left(a^{\dagger}(t) e^{\mathrm{i} \theta}+a(t) e^{-\mathrm{i} \theta}\right)$, where $a(t)$ is the Heisenberg output field operator. Thus the recorded data contain all information needed to extract the first order correlation function. This function is easily calculated using the input-output formalism for optical cavities [3].

$$
\begin{aligned}
g_{(1)}(\tau) & \equiv \frac{\left\langle a^{\dagger}(\tau) a(0)\right\rangle}{\left\langle a^{\dagger}(0) a(0)\right\rangle} \\
& =\frac{\left\langle x_{\theta}(\tau) x_{\theta}(0)\right\rangle_{\theta}}{\left\langle x_{\theta}(0) x_{\theta}(0)\right\rangle_{\theta}} \\
& =\frac{1-d^{2}}{2 d}\left[\frac{1}{1-d} e^{-(1-d) \Gamma \tau}-\frac{1}{1+d} e^{-(1+d) \Gamma \tau}\right] .
\end{aligned}
$$

Here $<>_{\theta}$ means averaging over all phase angles. Fig. 5 shows that the evaluation of the first order correlation function obtained from the experimental data is in good agreement with theory. In principle it is possible to obtain via broadband homodyne detection all higher order time correlations of the field quadratures $\left\langle x_{\theta}\left(\tau_{1}\right) x_{\theta}\left(\tau_{2}\right) \ldots x_{\theta}\left(\tau_{n}\right)\right\rangle$. Except for one special case [11] their significance does not appear to have been studied. For our data the evaluation of these correlations could not be done reliably, due to difficulties with a proper normalization at $\tau=0$ and since the minimum recordable timestep of $1.7 \mathrm{~ns}$ was too large in comparison to the expected rapid exponential decay times $e^{-n(1-d) \Gamma \tau}$.

Note also that since a homodyne measurement is always a two-mode measurement of the pair of modes at frequencies $\omega \pm \Omega$, our experiment shows two-mode time correlations. For single-mode 
time correlation measurements a heterodyne system (detection efficiency $\eta<0.5$ ) can be employed. This is possible, since detection efficiency does not play the same crucial role in time correlation measurements that it does for quantum state reconstructions, in fact ideally $g_{(n)}$ is independent of $\eta$.

In conclusion, we have demonstrated the first simultaneous reconstruction of a whole spectrum of quantum states of the optical light field. Some of the reconstructed states emitted from the OPA display photon number oscillations and strongly elliptical Wigner functions. These characteristics vanish for the mode-pairs at frequencies sufficiently away from the OPA cavity center frequency. Furthermore the first order time correlation function was evaluated in good agreement with theory.

Our measurement scheme may be also useful for a variety of other quantum optical systems with more complex frequency or time dependencies. Examples are recent squeezing experiments with solitons in a fibre [12], pump-noise-suppressed laser diodes [13], or exciton-polariton systems in semi-conductors 14, 15.

We thank Robert Bruckmeier and Kazimierz Rzążewski for very helpful discussions. Financial support was provided by the Deutsche Forschungsgemeinschaft and the Esprit LTR project 20029ACQUIRE. One of us (F.I.) also gratefully acknowledges financial support from the Alexander von Humboldt Foundation, and the hospitality of the LS Mlynek at the Fakultät für Physik, Universität Konstanz, while on leave of absence from the Dipartimento di Fisica, Università di Salerno.

[1] K. Vogel and H. Risken, Phys. Rev. A 40, 2847 (1989); D.T. Smithey, M. Beck, M.G. Raymer, A. Faridani, Phys. Rev. Lett. 70, 1244 (1993); see also special issue on Quantum State Preparation and Measurement, J. Mod. Opt. 44 number 11/12 (1997), or the review article by M. Freyberger, P. Bardroff, C. Leichtle, G. Schrade, and W. Schleich, Phys. World, Nov. 1997;

[2] G. Breitenbach, S. Schiller, and J. Mlynek, Nature, 387, 471 (1997); G. Breitenbach and S. Schiller, J. Mod. Opt. 44, 2207 (1997);

[3] M. J. Collett and D. F. Walls, Phys. Rev A 32, 2887 (1985); C.W. Gardiner, Quantum Noise, Springer, Berlin 1991; D.F. Walls, G.J. Milburn, Quantum Optics, Springer Berlin (1994);

[4] Z.Y. Ou and H.J. Kimble, Phys. Rev. A 52, 3126 (1995); M.G. Raymer, D.F. Mc Alister, and U. 
Leonhardt, Phys. Rev. A 54, 2397 (1996); Th. Richter, J. Mod. Opt. 44, 2385 (1997); H. Paul, P. Törmä, T. Kiss, and I. Jex, J. Mod. Opt. 44, 2395 (1997); T. Opatrný, D.-G. Welsch, and W. Vogel, Opt. Comm 134, 112 (1997); M.G. Raymer, D.T. Smithey, M. Beck, M. Anderson, and D.F. McAlister, Proc. III. intern. Wigner Symp., Oxford 1993, to appear in J. of Mod. Physics B;

[5] D.F. Mc Alister and M.G. Raymer, Phys. Rev. A 55, R1609 (1997);

[6] G. M. D'Ariano, C. Macchiavello, and M. G. A. Paris, Phys. Rev. A 50, 4298 (1994); U. Leonhardt, M. Munroe, T. Kiss, Th. Richter, and M.G. Raymer, Opt. Comm. 127, 144 (1996);

[7] G. Breitenbach, T. Müller, S. Pereira, J.-P. Poizat, S. Schiller und J. Mlynek, J. Opt. Soc. Am. B, 12, 2304 (1995); S. Schiller, G. Breitenbach, S. F. Pereira, T. MAller, and J. Mlynek, Phys. Rev. Lett. 77, 2933 (1996);

[8] G.M. D’Ariano, U. Leonhardt, and H. Paul, Phys. Rev. A 52, R1801 (1995); T. Kiss, U. Herzog, and U. Leonhardt, Phys. Rev. A 52, 2433 (1995);

[9] S.M. Tan, J. Mod. Opt. 44, 2233 (1997);

[10] R.E. Slusher, L.W. Hollberg, B. Yurke, J.C. Mertz, and J.F. Valley, Phys. Rev. Lett. 55, 2409 (1985); L.-A. Wu, H. J. Kimble, J. L. Hall and H. Wu, Phys. Rev. Lett. 57, 691 (1986); E. S. Polzik, J. Carri, and H. J. Kimble, Phys. Rev. Lett. 68, 3020 (1992);

[11] L. Mandel and E. Wolf, Optical coherence and quantum optics, chapt. 21, Cambridge University Press, Cambridge 1995;

[12] S.R.. Friberg, S. Machida, M.J. Werner, A. Levanon, and T.Mukai, Phys. Rev. Lett. 77, 3775 (1996); S. Spälter, M. Burk, U. Strössner, M. Böhm, A. Sizmann, and G. Leuchs, Europhys. Lett. 38, 335 (1997);

[13] C. Becher, E. Gehrig, and K.J. Boller, to appear in Phys. Rev. A;

[14] S. Jiang, S. Machida, Y. Takiguchi, H. Cao, and Y. Yamamoto, Opt. Comm. 145, 91 (1998);

[15] D. Boggavarapu, D. McAlister, M. E. Anderson, M. Munroe, M. G. Raymer, G. Khitrova, and H. Gibbs, Proceedings of Quantum Electronics and Laser Science Conference (June 2-7, 1996, Opt. Soc. of Am., Technical Digest Vol.9, 1996), pg.33.; M. E. Anderson, M. Munroe, U. Leonhardt, D. 
Boggavarapu, D. F. McAlister and M. G. Raymer, Proceedings of Generation, Amplification, and Measurement of Ultrafast Laser Pulses III, pg 142-151 (OE/LASE, San Jose, Jan. 1996) (SPIE, Vol. $2701,1996)$

[16] R. Loudon, The quantum theory of light, Oxford 1973;

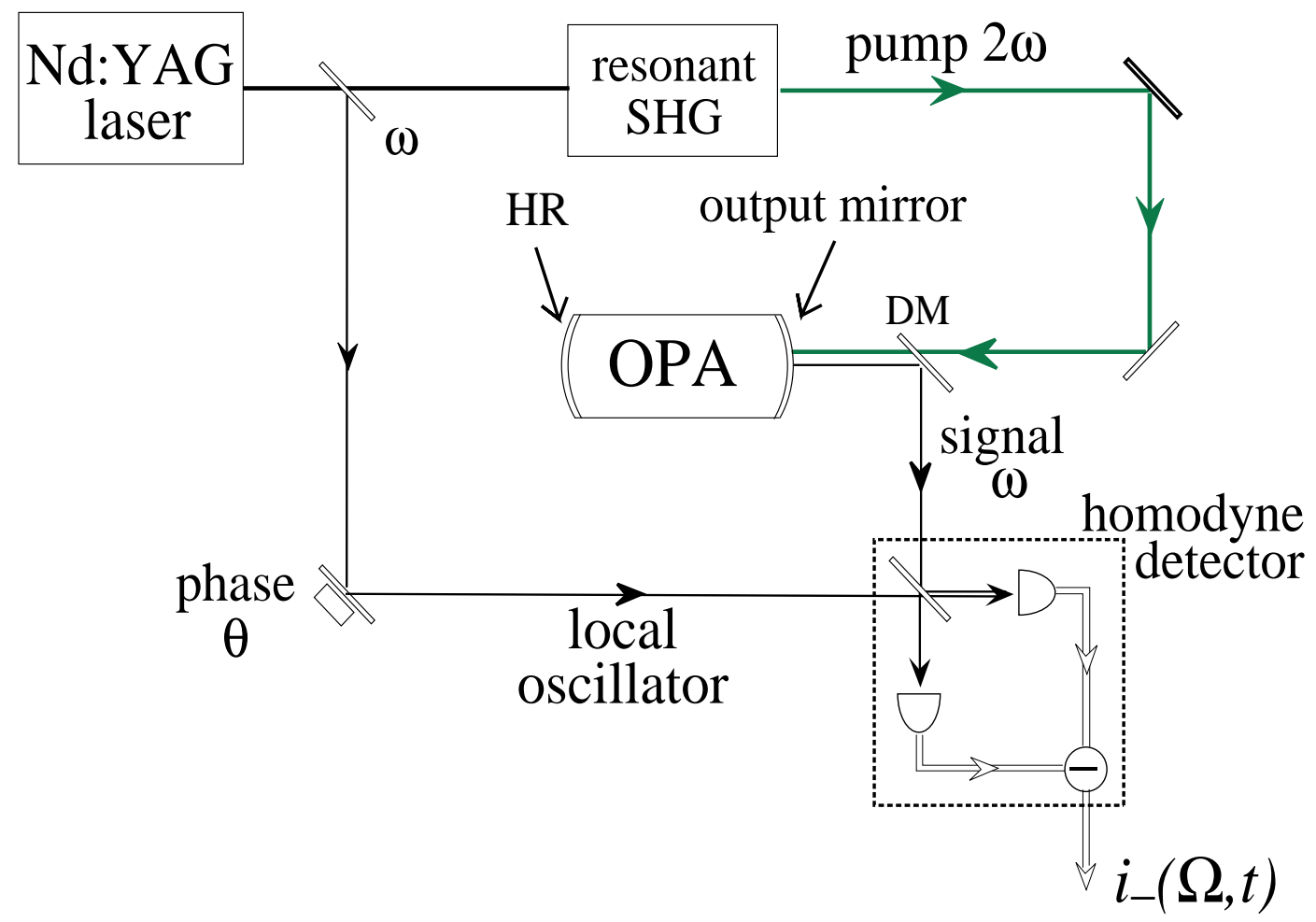

FIG. 1. Experimental scheme for measuring the quantum states of the OPA. 


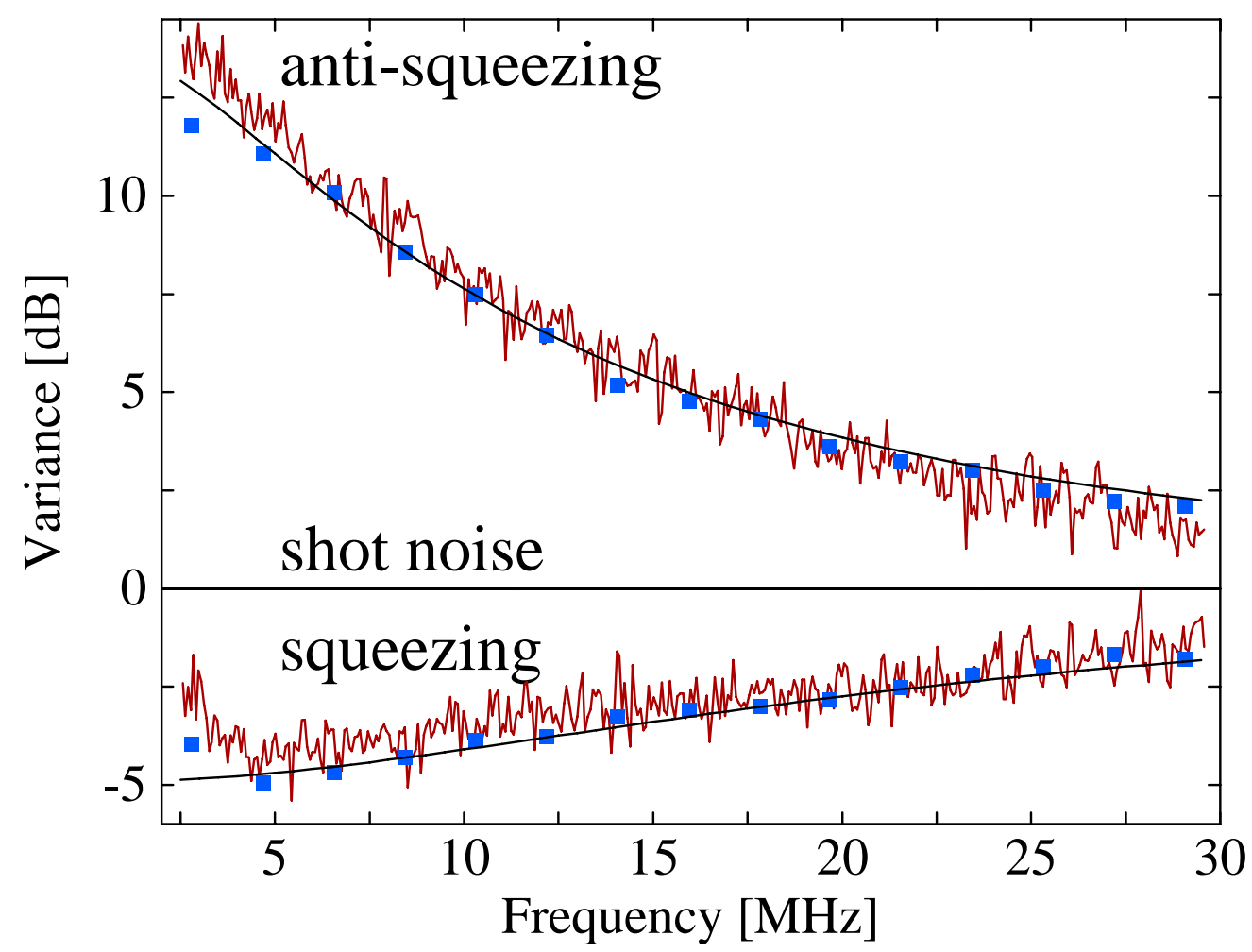

FIG. 2. Spectrum of squeezing and anti-squeezing: Spectrum analyzer traces (upper and lower grey lines) in comparison with a theoretical fit (smooth black lines) using eq.1. Each of the 15 pairs of black squares corresponds to one reconstructed quantum state at a specific frequency $\Omega$ and represents the maximum/minimum width of the sampled marginal distributions. The reduction in squeezing at frequencies below 4.5 MHz is due to classical laser noise at low frequencies. 


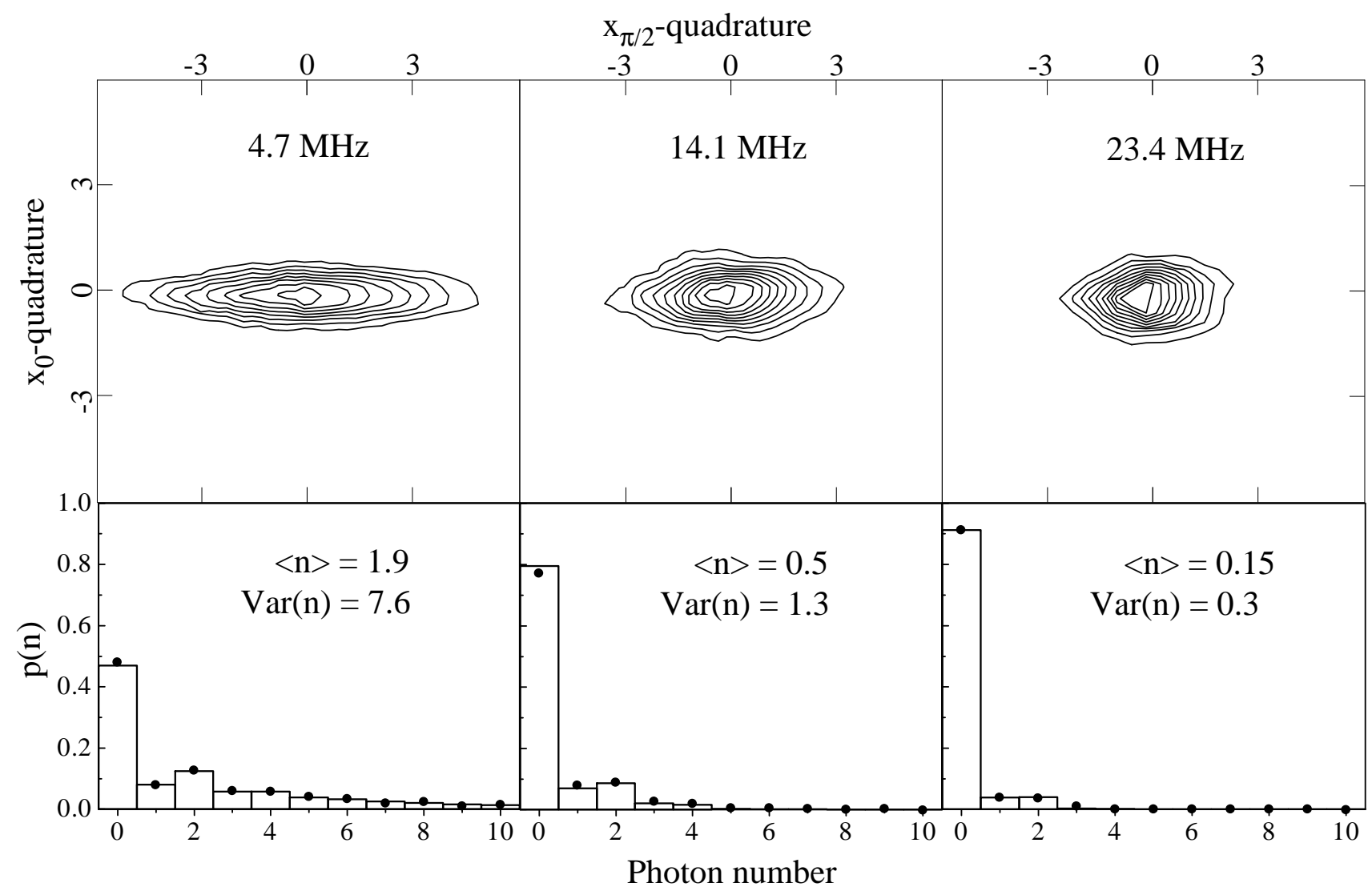

FIG. 3. Three examples of the simultaneously recorded squeezed vacuum states emitted by the OPA: Upper row: contours of the Wigner functions, lower row: corresponding reconstructed photon number distributions (dots) with theoretical expectations (histograms). For increasing offset frequency $\Omega$ from the OPA cavity center frequency the states aproach the vacuum state and the characteristics of squeezing such as photon number oscillations and ellipticity of the Wigner function vanish. 


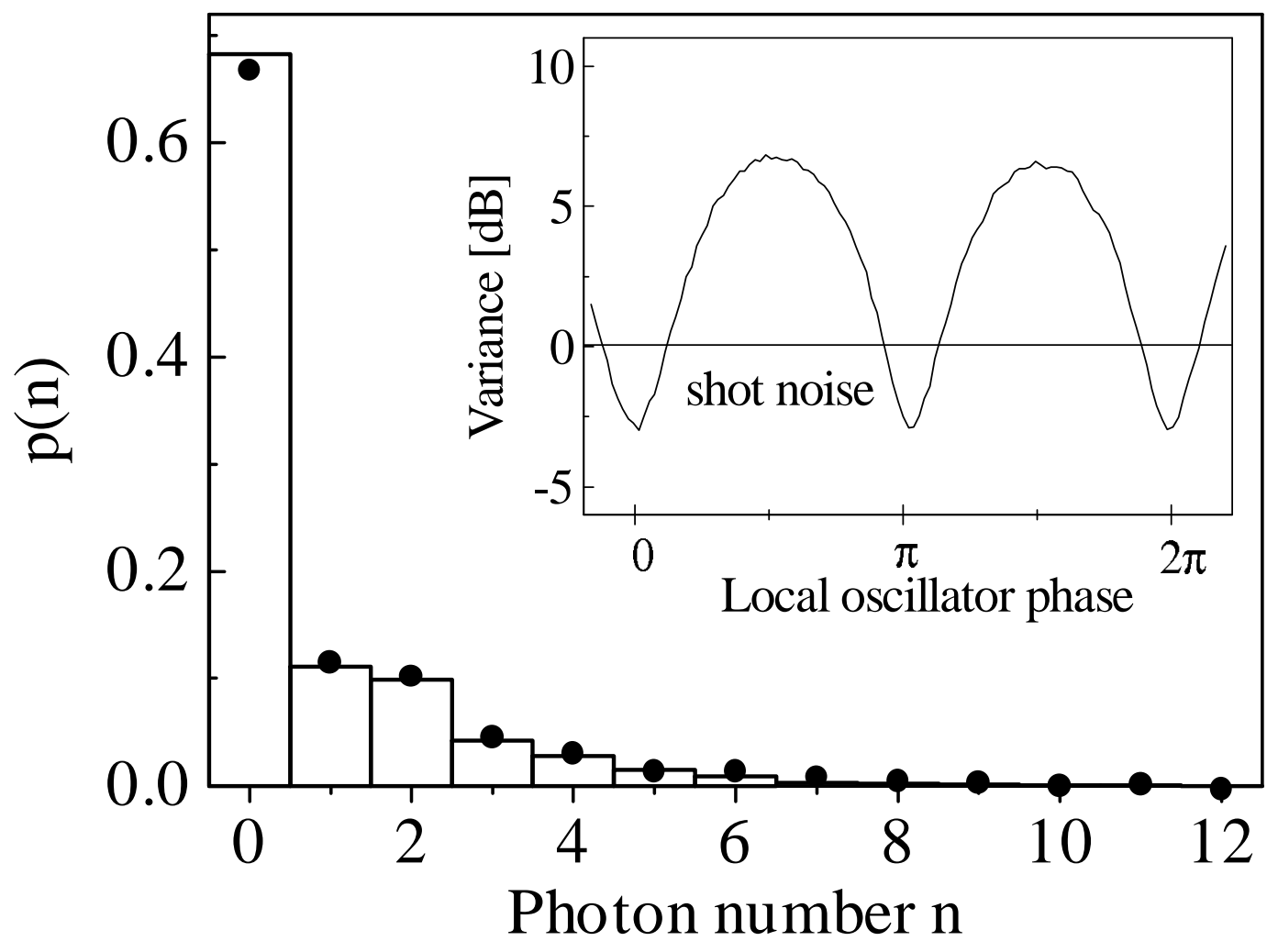

FIG. 4. Electric field variances in dependence of the local oscillator phase and photon statistics of the sum of all OPA modes between 2 and $30 \mathrm{MHz}$. The average photon number $\langle n\rangle=0.8$ gives the average photon flux per $\mathrm{Hz}$ bandwidth. This implies a total photon flux of $2.2 \cdot 10^{8}$ photons $/ \mathrm{s} \approx 45 \mathrm{pW}$ within the detection bandwidth of $28 \mathrm{MHz}$. 


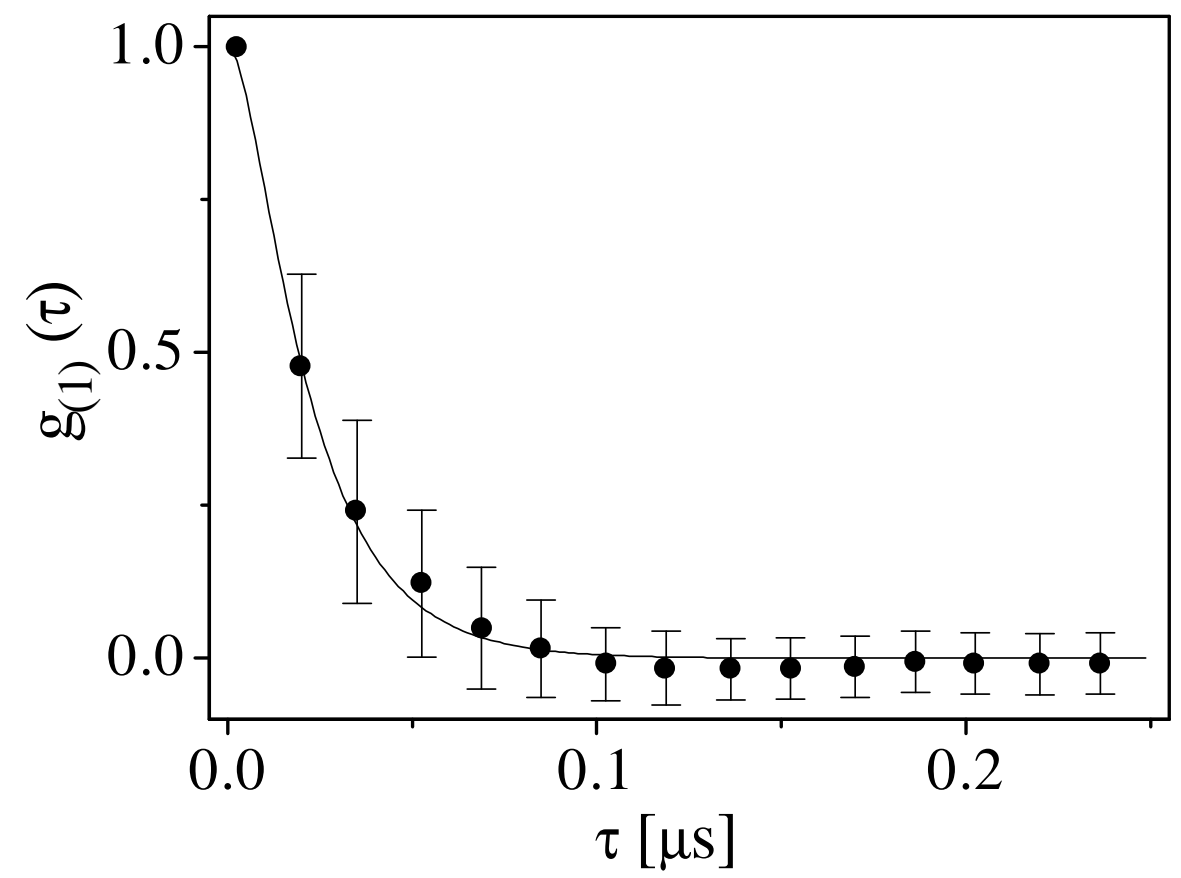

FIG. 5. First--order time correlation function of the recorded noise trace (dots) in comparison with theory (line). A quasi-exponential decay similar to the one of a thermal state is observed. 